\title{
Phenomenological description of phase inversion
}

\author{
K. Piela and G. Ooms* \\ J.M. Burgerscentrum, Laboratory for Aero- and Hydrodynamics, Delft University of Technology, Leeghwaterstraat 21, 2628 CA Delft, \\ The Netherlands \\ J. V. Sengers \\ Burgers Program for Fluid Dynamics, Institute for Physical Science and Technology, University of Maryland, College Park, \\ Maryland 20742, USA
}

(Received 19 May 2008; revised manuscript received 28 November 2008; published 10 February 2009)

\begin{abstract}
We propose an extended Ginzburg-Landau model for a description of the ambivalence region associated with the phenomenon of phase inversion observed in dispersed water-oil flow through a pipe. In analogy to the classical mean-field theory of phase transitions, it is shown that a good quantitative representation of the ambivalence region is obtained by using the injected phase volume fraction and a friction factor as the appropriate physical parameters.
\end{abstract}

DOI: 10.1103/PhysRevE.79.021403

PACS number(s): 82.70.- $-\mathrm{y}, 47.55 .-\mathrm{t}$

\section{INTRODUCTION}

The flow of two immiscible liquids often occurs as a dispersed flow, where one liquid is present in the other one in the form of drops. Dispersions are widely used in the petrochemical, food, chemical, and pharmaceutical industries. Handling and controlling dispersion properties is of key interest for practical applications. Oil-water dispersions can occur as oil drops in a water continuous phase or water drops in an oil continuous phase. Phase inversion is the phenomenon by which the dispersed phase becomes the continuous one, and vice versa. During this process the effective viscosity of the mixture becomes very large, which leads to a high pressure drop or low flow rate. Prediction of the critical concentration where phase inversion occurs, is important for two main reasons. It can help in designing processes in such a way, that a high-pressure buildup due to inversion is avoided. It can also help in designing processes, in which a dense dispersion is necessary for achieving the correct product property (for instance, in the food or pharmaceutical industry).

The phase-inversion phenomenon has been studied for many years (see, for instance, $[1,2])$. Several models proposed in the literature for predicting the critical concentration are based on a description of a balance between breakup and coalescence of the drops forming the dispersed phase (see, for instance, $[3,4]$ ). In $[5,6]$ it is assumed that inversion occurs, when the surface energy of the oil-in-water dispersion is equal to the surface energy of the water-in-oil dispersion and the surface energy is calculated based on the drop size which is again calculated from a balance between breakup and coalescence.

Experimentally it has been found that a so-called ambivalence region exists, in which both phases may be found to be continuous. Only outside this region one phase is always continuous and the other is always discrete. Inside the ambivalence region either one of the two phases can be continu-

\footnotetext{
*g.ooms@tudelft.nl
}

ous and the exact value at which phase inversion occurs depends on the operating conditions, surface tension, pipe wettability, and chemical properties. Based on some of the aforementioned models attempts have been made to predict the ambivalence region. For instance, in [5], an attempt has been made to predict the ambivalence region by calculating the drop size using a different relation for an oil-in-water dispersion than for a water-in-oil dispersion. In [6] it was suggested that a possible reason for the hysteresis (ambivalence) region is the time needed after phase inversion for the new continuous phase to completely wet the wall.

Most of the experiments reported in the literature were performed in a stirred vessel and usually water and oil were used. They were often "continuous experiments" during which the dispersed phase was gradually added to the continuous phase. For this type of experiments it was found, that phase inversion could be postponed to a high value $(>0.8)$ of the dispersed phase volume fraction. Also a wide ambivalent volume-fraction region existed where the mixture could be either water continuous or oil continuous [4,7-10]. Alternatively, during "direct experiments" in a stirred vessel the two liquids were mixed at a certain concentration $[11,12]$ and inversion usually occurred at a value of the dispersed phase fraction close to 0.5 (dependent on the properties of the liquids) and no ambivalence region was observed.

Only a few phase-inversion experiments have been carried out in a pipe. Direct experiments in a vertical pipe have been reported in [13] and direct experiments in a horizontal pipe in [14-17]. In these experiments particular attention was paid to the pressure-drop increase over the pipe during phase inversion. Recently, we have carried out continuous phase-inversion experiments in a pipe [18]. During these experiments we started with a flow of one of the liquids and gradually injected the other liquid, while keeping the mixture velocity constant. We measured also a strong increase in the pressure drop during the inversion process. Depending on the injected phase volume fraction $\chi$ (the ratio between the injection rate of the dispersed phase and the flow rate of the mixture in the pipe), it was found that phase inversion could be postponed up to high values of the dispersed phase. With decreasing values of $\chi$ the critical concentration increased, 


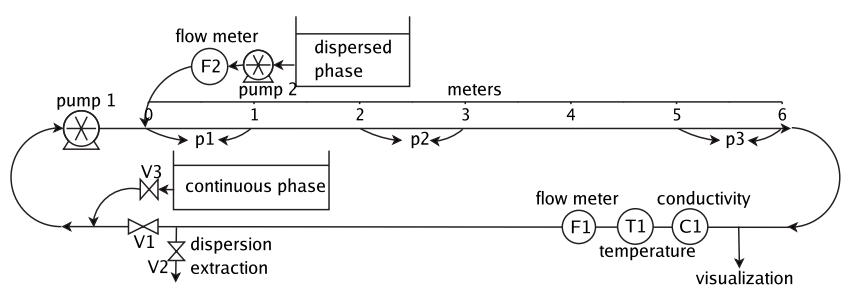

FIG. 1. Sketch of the experimental setup for continuous experiments.

even up to values of 0.9 . Hence, the ambivalence region for these experiments was very wide at small values of $\chi$ and it became narrower with increasing $\chi$. At a value of about $\chi$ $=0.5$ the ambivalence region disappeared and the critical concentration became equal to the one found during direct experiments (see [19]), whereby the two liquids are injected from the start simultaneously into the pipe with certain concentrations.

It is not evident to us, how this important result from the experiments reported in [18] can be explained with one of the aforementioned models. Therefore, we have developed a framework for the interpretation of the phase-inversion experiments adopting an analogy with the classical GinzburgLandau mean-field theory of phase transitions in thermodynamics [20]. By considering the friction factor $f$ as function of the injected phase volume fraction $\chi$, we have succeeded to obtain a realistic description of the ambivalence region for a range of conditions with different values of the injected phase volume fraction during continuous experiments.

In Sec. II we briefly review our experimental results $[18,19]$. In Sec. III we adopt a Ginzburg-Landau model for the interpretation of our phase-inversion experiments. In Sec. IV it is shown how this model yields a quantitative representation of the ambivalence region by using the injected phase volume fraction $\chi$ and a measured friction factor $f$ as the characteristic physical variables of the phase-inversion process. In Sec. V we show how the model can be used in the absence of experimental data for the friction factor by relating the measured friction factor $f$ to an effective viscosity. Our results are summarized in Sec. VI.

\section{EXPERIMENTAL OBSERVATIONS}

In our experimental studies [18,19] many phase-inversion experiments were performed on an oil-water flow through a pipe. Two types of experiments were performed: continuous experiments and direct experiments.

The sketch of the setup used for the continuous experiments is shown in Fig. 1. The symbols used in the sketches have the following meaning: V, valves; F1, Krohne Optimass 7000 flow meter (measuring error $<0.26 \%$ ); F2, Krohne Corimass E flow meter (measuring error $<0.4 \%$ ); $\mathrm{C} 1$, conductivity cell; and T1, thermocouple. During the continuous experiments one of the liquids (water or oil) was taken from the continuous phase tank (see Fig. 1) and recirculated through the pipe loop by means of pump 1. After recirculating the liquid for a few minutes to ensure that the pipe walls were wetted by the liquid, injection of the other liquid started by pumping (using pump 2) the dispersed phase through the injector into the pipe loop. During the injection, valve 2 was opened and the same volume of dispersion liquid was removed from the pipe loop as the volume that was injected. Flow meter 1 measured the density and the flow rate of the mixture in the pipe loop. The mixture velocity was kept constant during the experiment by an electronic feedback system (pump 1 was controlled based on measurements of flow meter 1).

During continuous experiments the dispersed phase was gradually added to the flowing mixture in the closed pipe loop. The dispersed phase volume fraction was slowly increased and at a certain moment inversion occurred. During these experiments it was noticed that the dispersed phase fraction at which inversion occurs, depends nearly linearly on the injected phase volume fraction $\chi$. By starting an experiment with pure water and gradually adding oil and by starting with pure oil and gradually adding water the ambivalence region was measured. It was found that the ambivalence region is almost independent of the Reynolds, Froude, and Weber numbers and also independent of the injection velocity of the dispersed phase [as long as the mixture velocity is sufficiently large $(\geqslant 2 \mathrm{~m} / \mathrm{s})]$. However, it was found that the injection phase volume fraction $\chi$ had a significant influence on the width of the ambivalence region. As can be seen in Table I all experiments were conducted at high Re, We, and Fr numbers. According to [21] a mixture velocity of $2 \mathrm{~m} / \mathrm{s}$ is sufficiently large to be sure of a dispersed pipe flow during our experiments.

For direct experiments the setup shown in Fig. 1 was modified. The pipe loop was opened and two additional straight pipe sections were added. During the direct experiments, the two liquids were injected (from separate tanks) simultaneously into the pipe by means of pump 1 and pump 2. The two liquids flowed through the pipe to the large separation tank, which was situated at the end of the last pipe section. The mixture was injected at a constant concentration for at least $40 \mathrm{~s}$. Flow meter 1 measured the density and the flow rate of the mixture in the pipe. The pressure gradient was measured at six different positions: Immediately downstream of the inlet and at distances of $2.0 \mathrm{~m}(125 d), 5.0 \mathrm{~m}$ $(313 d), 11.7 \mathrm{~m}(731 d), 18.7 \mathrm{~m}(1169 d)$, and $26.5 \mathrm{~m}(1656 d)$ from the inlet, where $d$ is the pipe diameter.

The phase-inversion process was determined by the mixing between oil and water at and after the inlet. At the critical concentration, water-continuous, and oil-continuous regions were created simultaneously. These regions interact while being transported downstream by the flowing mixture. The interaction was very similar to the one observed during the phase-inversion process of the continuous experiments. The direct experiments can be considered as limiting cases of continuous experiments, with a large injection phase volume fraction of the dispersed phase and with inversion taking place before one cycle through the pipe loop is completed. No ambivalence region was observed during the direct experiments. In the case of direct experiments the injected phase volume fraction is calculated as the ratio of the flow rate of the liquid with the lowest volume fraction and the flow rate of the mixture in the pipe.

The observed ambivalence region for the case of continuous experiments is shown in Fig. 2. As can be seen from this 
TABLE I. Dimensionless numbers for water-continuous or oil-continuous experiments at different superficial velocities. (The superficial velocity of a certain fluid is defined as the ratio of the volume flow rate of that fluid and the pipe cross section.) For the calculation of the Froude number the density difference between water and oil is used and for the calculation of the Weber number the interfacial tension between the two liquids. "Dispersed phase boundary" indicates the transition from the dispersed flow pattern to another flow pattern according to [21]

\begin{tabular}{|c|c|c|c|c|}
\hline Water & $\mathrm{Re}$ & $\mathrm{Fr}$ & We & \\
\hline $1 \mathrm{~m} / \mathrm{s}$ & $1.6 \times 10^{4}$ & 6.4 & 354 & \\
\hline $2 \mathrm{~m} / \mathrm{s}$ & $3.2 \times 10^{4}$ & 25.5 & 1418 & \\
\hline $3 \mathrm{~m} / \mathrm{s}$ & $4.8 \times 10^{4}$ & 57.3 & 3190 & \\
\hline $1.34 \mathrm{~m} / \mathrm{s}$ & $2.1 \times 10^{4}$ & 11.4 & 637 & dispersed phase boundary $[21]$ \\
\hline Shell macron EDM 110 & $\mathrm{Re}$ & Fr & We & \\
\hline $2 \mathrm{~m} / \mathrm{s}$ & $0.7 \times 10^{4}$ & 25.5 & 1129 & \\
\hline $3.5 \mathrm{~m} / \mathrm{s}$ & $1.1 \times 10^{4}$ & 78 & 3458 & \\
\hline $1.35 \mathrm{~m} / \mathrm{s}$ & $0.4 \times 10^{4}$ & 11.6 & 515 & dispersed phase boundary [21] \\
\hline
\end{tabular}

figure, the width of the ambivalence region depends strongly on the injected phase volume fraction $\chi$. The experimental results lie on two lines, one for the water-to-oil experiments and one for the oil-to-water experiments. [Two experimental data points for the oil-to-water experiments do not coincide with the line. The explanation for this deviation was given in [18]: Entrapment of the continuous phase (oil) into the dispersed phase (water) increases the effective dispersed phase fraction. If the concentration of oil present as oil droplets inside the water drops would be subtracted from the total oil concentration, the experimental data points would fit the line. These two points will be omitted in the remainder of this presentation.]

In Fig. 3 the friction factor $f$ (calculated from the measured pressure drop by means of $f=2 \Delta P d / \rho u^{2} L$, where $\Delta P$ is the pressure drop over a distance $L, d$ the pipe diameter, $\rho$

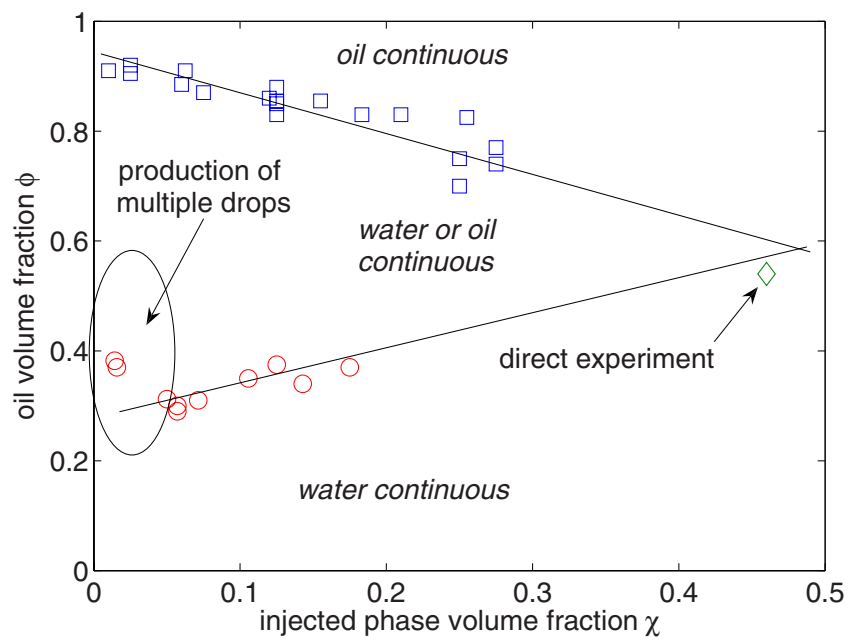

FIG. 2. (Color online) Inversion map. The upper line gives the oil volume fraction at the point of inversion as measured during water-to-oil continuous experiments. The lower line represents the oil volume fraction at inversion as measured during oil-to-water continuous experiments. The region in between the two lines is the ambivalence region, where both oil and water can be the continuous phase. Also the oil volume fraction at inversion as measured during the direct experiments is indicated. the density and $u$ the average mixture velocity) is shown as function of the oil volume fraction $\phi$ for water-to-oil continuous experiments presented in Fig. 2. As can be seen, the friction factor $f$ strongly increases when the concentration of the dispersed phase exceeds 0.6. The friction factor does not depend on the injected phase volume fraction prior to the occurrence of phase inversion. (Although the critical concentration at which phase inversion occurs, depends on $\chi$.) The friction factor was also found to be independent of the mixture velocity. A similar behavior was observed for oil-towater continuous experiments.

Figure 4 shows the different stages of the phase-inversion process during a continuous experiment of a watercontinuous flow to an oil-continuous flow (water-to-oil experiment) for a mixture velocity of $1 \mathrm{~m} / \mathrm{s}$ and an injection phase volume fraction $\chi=0.125$. The left-hand picture shows the start of the inversion process at an oil volume fraction $\chi=0.84$. Some larger oil multiple drops (containing water droplets) are formed in a water-continuous region due to coalescence of the original oil drops. After $16 \mathrm{~s}$ (second picture) the number of these oil-continuous pockets has increased due to continuous oil injection via the injector. With further oil

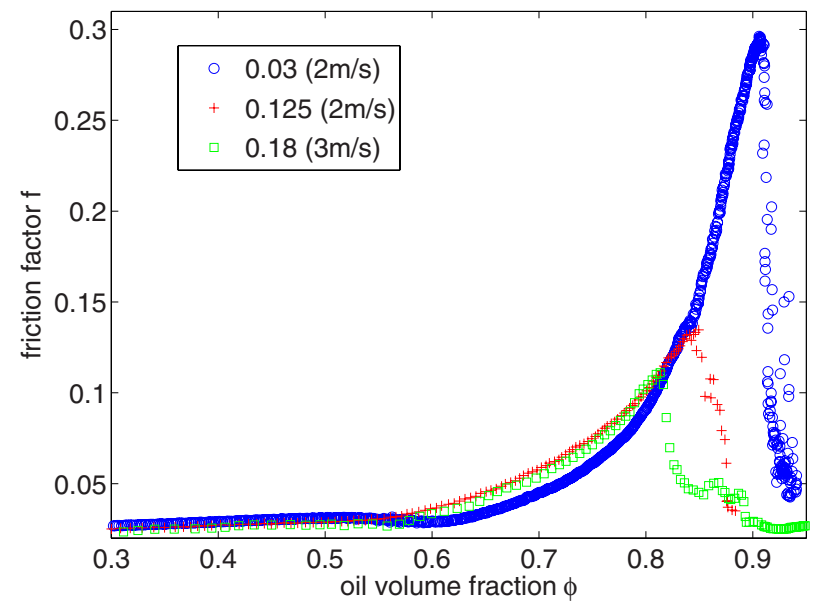

FIG. 3. (Color online) Friction factor for three water-to-oil experiments at a mixture velocity of $2 \mathrm{~m} / \mathrm{s}$ and $3 \mathrm{~m} / \mathrm{s}$ and different values of the injected phase volume fraction $(0.03,0.125,0.18)$. 


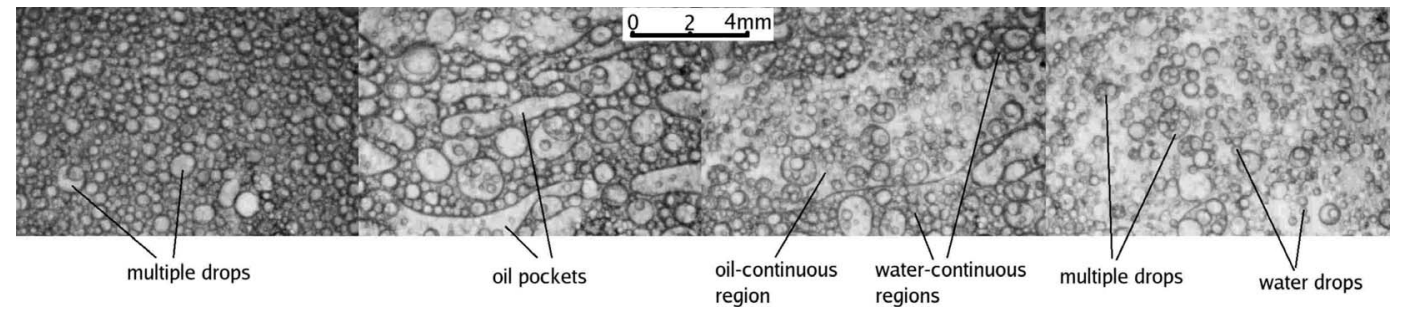

FIG. 4. Inversion from a water-continuous to an oil-continuous flow during a continuous phase-inversion experiment.

injection, isolated water-continuous pockets are created embedded in oil-continuous regions. After $43 \mathrm{~s}$ (third picture) the oil-continuous pockets in water-continuous regions and water-continuous pockets in oil-continuous regions are about the same in number and size. With further oil injection, the oil-continuous pockets disappear and only water-continuous pockets in an oil-continuous region remain. After $87 \mathrm{~s}$ (righthand picture) the phase inversion is completed and the mixture is oil-continuous with water drops.

During a direct experiment the phase fractions were kept constant in time. Since the two phases are well mixed the dispersed phase volume fraction in the pipe is equal to the dispersed phase volume fraction at the point of injection at the entrance to the pipe loop. Close to the point where the flow changed from a water-continuous to an oil-continuous flow or vice versa, strong variations in the morphological structures of the dispersion were observed. For a mixture velocity of $2 \mathrm{~m} / \mathrm{s}$, these changes occur at oil volume fractions $\phi$ between 0.5 and 0.6. Figure 5 shows the dispersion morphology for two different values of the oil volume fraction. At $\phi=0.56$ (see left-hand picture) the flow consists of many (water-continuous) pockets in oil-continuous regions (dark regions), and (multiple) oil drops in water-continuous regions (see, for instance, the center part of the picture). At $\phi=0.58$ (see right-hand picture) most of the flow field is oil continuous.

There is an important difference between a continuous experiment and a direct experiment. In a continuous experiment the inversion process starts with the coalescence of drops leading to the formation of larger drops, pockets, and regions by encapsulation of parts of the continuous phase. Finally this process causes the disappearance of regions of the originally continuous phase. In a direct experiment the two liquids are mixed from the start at a constant concentration of the phases and there is no preliminary structure. Depending on the concentration, the liquids mix as a watercontinuous mixture or an oil-continuous one. However, at an oil volume fraction $\phi$ between 0.5 and 0.6 both watercontinuous and oil-continuous regions are created during the same experiment (because of the nonhomogeneous mixing, in particular in the T-junction and the entrance region of the pipe). At a value of $\phi$ between 0.5 and 0.6 , none of the regions is sufficiently strong to dominate the other one, and so both regions flow downstream. These regions interact, entrap parts of the other continuous phase, breakup, and coalescence causing a large pressure gradient over the pipe.

A more detailed description of the experimental observations of phase inversion during continuous and direct experiments can be found in some of our previous presentations
$[18,19]$. Pictures taken during phase inversion were presented and measurements of the pressure drop and conductivity were reported. The behavior of the pressure drop increase during inversion was explained by visual observations. Also interactions during phase inversion were described. These results showed that phase inversion during continuous and direct experiments, despite the different onset, has a very similar behavior.

\section{GINZBURG-LANDAU MODEL FOR PHASE INVERSION}

During phase inversion very complex structures are created (as described in [19]). Regions, pockets, and (multiple) drops interact with each other. The complexity of the structures and their interaction make it very difficult to model phase inversion in detail. However, we know from thermodynamics that phase transitions can be described by a meanfield approach without any need for a knowledge of the detailed molecular interactions. In this paper we propose a similar approach for a description of the phenomenon of phase inversion.

The mean-field theory of phase transitions is based on a classical Landau expansion of an appropriate mesoscopic potential $(G)$ in terms of an order parameter $s$. The simplest version is a two-term Ginzburg-Landau model of the form

$$
G(s)=G(s)=s^{4}+a s^{2},
$$

where the coefficient $a$ is a control parameter that is related to the difference between the actual temperature $T$ and a critical temperature $T_{c}$ [20,22]. This model yields a bifurcation at $a=0$ (i.e., at $T=T_{c}$ ) with a quadratic phase boundary asymptotically close to the critical point [23]. It is of interest to note that the Ginzburg-Landau potential given by Eq. (1) is symmetric in terms of the order parameter $s$ [24]. It has

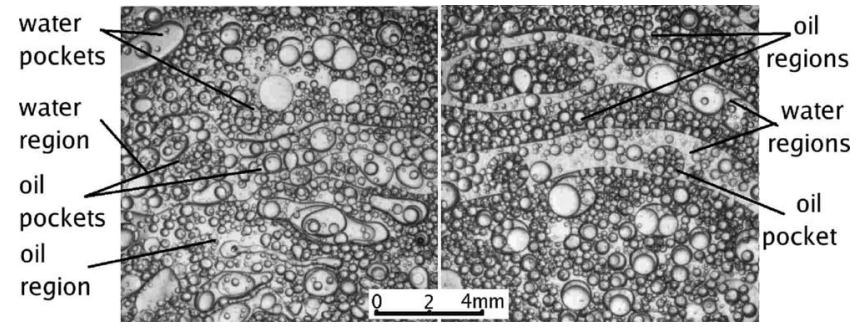

FIG. 5. Direct experiment at a mixture velocity $2 \mathrm{~m} / \mathrm{s}$. Pictures taken at different oil volume fractions (left-hand picture at $\phi$ $=0.56$, right-hand picture at $\phi=0.58$ ). 
been demonstrated recently that the actual asymmetries observed in phase transitions in fluids and fluid mixtures do not originate from any odd terms in the Ginzburg-Landau potential (1), but from the relationship between the order parameter $s$ and the physical densities, both for the mean-field theory of phase transitions which is the one relevant here [25], and for the nonclassical theory of phase transitions $[26,27]$. A major difference between equilibrium phase transitions and nonequilibrium phase transitions is that in nonequilibrium the phase transition is driven by an externally imposed field. In our problem the field driving the phase transition is the shear rate induced by the pressure drop. Such an external field will necessarily break the symmetry of the mesoscopic potential in terms of the order parameter and, hence, will induce in first approximation a term proportional to $s$. Thus to describe our phase-inversion experiments we need a slightly more general Ginsburg-Landau potential of the form $[28,29]$ :

$$
G(s)=s^{4}+a s^{2}+b s,
$$

where the second control parameter $b$ is the one driving the nonequilibrium phase transition which in the subsequent section will be identified with the friction factor $f$, which is proportional to the pressure drop. In this section we show that the mesoscopic potential Eq. (2) has all the ingredients to account for the phenomena observed in our phaseinversion experiments.

Depending on the values of $a$ and $b$ the $G(s)$ function has one or two minima. The location of the minima in the $G(s)$ domain are found by the following condition:

$$
\frac{d G}{d s}=4 s^{3}+2 a s+b=0
$$

and the number of minima changes from one to two when the second derivative of $G$ to $s$ becomes equal to zero,

$$
\frac{d^{2} G}{d s^{2}}=12 s^{2}+2 a=0 .
$$

Eliminating $s$ from Eqs. (3) and (4) leads to a relation between $a$ and $b$ for the change over from one to two minima

$$
(2 a / 3)^{3}+b^{2}=0 .
$$

The shape of this relation between $a$ and $b$ is shown in Fig. 6. Asymptotically the two branches merge according to a power-law behavior of the form $b \propto|a|^{3 / 2}$. Hence, Eq. (2) is also being referred to as a bifurcation model exhibiting a cusp catastrophe $[4,28,29]$.

When the line M-P-R-Q and the line N-Q-S-P in Fig. 6 are followed, the shape of the $G(s)$-function changes as sketched in Fig. 7. Let us start with the line M-P-R-Q. At point $M$ only one minimum in the free energy exists and the system is assumed to be in this minimum (indicated by the black spot in Fig. 7). Increasing $b$ in Fig. 6 moves the system to point $P$, where a second minimum starts to appear. At point $R$ there are two symmetric minima with a barrier between them, so the system remains in the left-hand minimum. At point $Q$ the barrier disappears and even a small perturbation causes the system to move to the right-hand

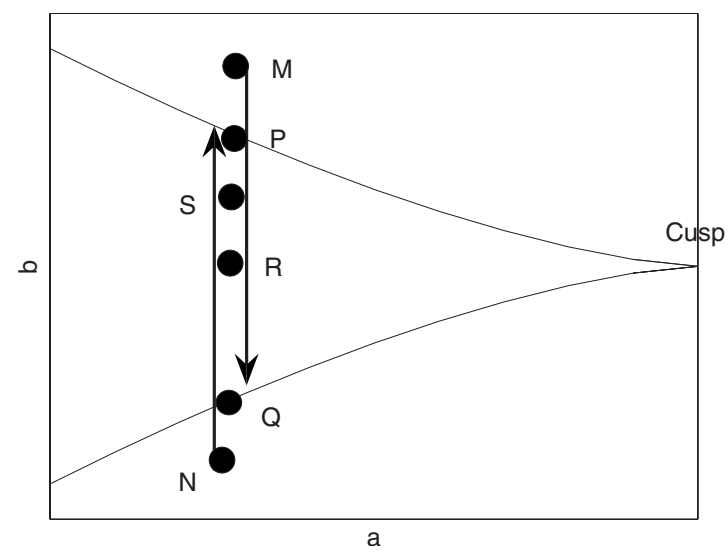

FIG. 6. Relation between $a$ and $b$ where a changeover takes place from one to two minima in the $G(s)$ function.

minimum. Similar behavior is observed for the line N-Q-S-P, which is followed with decreasing value of $b$. As can be seen a hysteresis occurs. When the line M-P-R-Q is followed, a transition from the left-hand minimum to the right-hand one takes place at point Q. When the line N-Q-S-P is followed, a transition from the right-hand minimum to the left-hand one occurs at point P. So between $\mathrm{P}$ and $\mathrm{Q}$ two states are possible and the actual state depends on the starting condition.

This behavior with a hysteresis effect based on the Ginzburg-Landau model, Eq. (2), is very similar to the physical behavior in the ambivalence region observed in our continuous experiments. Also Fig. 6, where two states are possible between the two converging lines, looks the same as the ambivalence region shown in Fig. 2. So the idea to use an approach for the description of phase inversion similar to the one used for the description of phase transitions looks attractive. In the next section this idea will be worked out in detail.

\section{APPLICATION TO PHASE INVERSION}

We submit that the injected phase volume fraction $\chi$ is a correct quantity to be used as a control parameter for the interpretation of the ambivalence region measured during phase-inversion experiments in terms of the hysteresis region of the Ginzburg-Landau model. With increasing $\chi$ the injec-

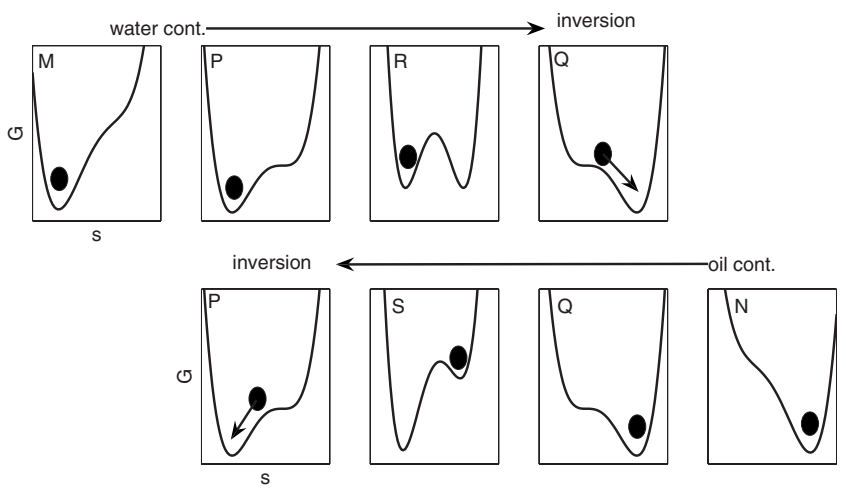

FIG. 7. Changes in the shape of the $G(s)$ function with passage through the line M-P-R-Q and the line N-Q-S-P in Fig. 6. 
tion rate of the dispersed phase into the mixture increases (at constant mixture velocity). This causes a stronger disturbance in the dispersion and the barrier in the $G(s)$ domain can more easily be taken. Therefore, with increasing $\chi$ the width of the ambivalence region will become smaller. Hence, we use $\chi$ as the first physical relevant parameter.

In systems described by the mesoscopic potential Eq. (2), $b$ represents an external ordering field that may be switched off. For a water-oil flow through a pipe this is the shear stress field caused by the external pressure drop over the pipe. As the correlating quantities should be nondimensional, this leads to the friction factor $f$ (nondimensional pressure drop) as the second physical relevant parameter.

The order parameter $s$ in the extended Ginzburg-Landau potential Eq. (2) represents the possible mesoscopic conditions of the liquids during their flow through the pipe. With mesoscopic conditions, we mean their mutual distribution (size of drops, presence of multiple drops, etc.). The resulting macroscopic condition will correspond to a minimum of $G(s)$ as function of $s$. To calculate $G(s)$ as a function of $s$ explicitly is difficult. However, as also noted by previous investigators [29], the nice property of the Ginzburg-Landau model is that it gives an explicit relation, Eq. (5), for the control parameters associated with the macroscopic states of the system that will enable us to characterize the ambivalence region in terms of only two system-dependent parameters.

As mentioned in Sec. II, we have observed an ambivalence region with a cusp. At the termination of the cusp (in Fig. 6) $a=0$. The continuous experiments show that the width of the ambivalence region decreases with increasing value of $\chi$. The ambivalence region even disappears at about $\chi=0.5$. This makes sense, as at $\chi=0.5$ it does not matter which liquid is in the continuous phase and which liquid is in the dispersed one. So the cusp in the ambivalence region is at about $\chi=0.5$. Hence, we assume the following linear relation between $a$ and $\chi$,

$$
a=\chi-0.5 \text {. }
$$

The relation between $b$ and $f$ is assumed to have the following form:

$$
b=K_{b 1}\left(f-K_{b 2}\right)
$$

in which $K_{b 1}$ and $K_{b 2}$ are constants. To determine the values of these constant two experimental data points $(A$ and $B$ ) were fitted to Eq. (5) implied by the Ginzburg-Landau model. During a water-to-oil experiment with an injection phase volume fraction of $\chi_{A}=0.05$ phase inversion took place at an oil volume fraction of $\phi_{A}=0.9$ and during an

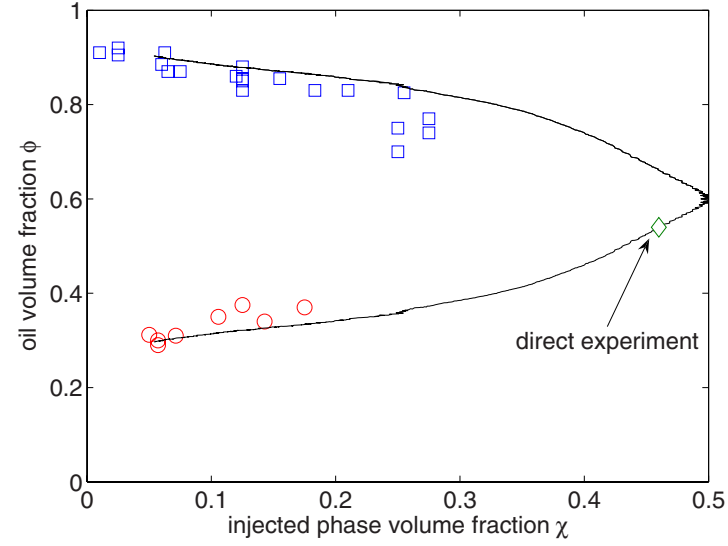

FIG. 8. (Color online) The oil volume fraction $\phi$ as a function of the injected phase volume fraction $\chi$ at the boundaries of the ambivalence region. The circles and squares indicate the experimental data points and the curves represent the values calculated from the Ginzburg-Landau model.

oil-to-water experiment with a $\chi_{B}=0.05$ phase inversion occurred at $\phi_{B}=0.31$. These two points $A(\chi=0.05, \phi=0.9)$ and $B(\chi=0.05, \phi=0.31)$ (and their related $f$ values) were selected to determine $K_{b 1}$ and $K_{b 2}$.

Once the constants have been determined, substitution of Eqs. (6) and (7) into Eq. (5) gives the ambivalence region in the $(\chi, f)$ domain. Using the experimentally found relation between $\phi$ and $f$, we can also calculate the ambivalence region in the $(\chi, \phi)$ domain. The result is shown in Fig. 8. As can be seen the calculated ambivalence region agrees well with the experimental data. Also the value from the direct experiment is on the calculated line. (The curve in Fig. 8 does not terminate in the cusplike behavior as in Fig. 6, since the conversion from $f$ to $\phi$ distorts the curve.)

We used the experimental data points $\mathrm{A}$ and $\mathrm{B}$ to determine the constants $K_{b 1}$ and $K_{b 2}$, since these points have the highest accuracy. To investigate the sensitivity of the description to the choice of pair of data points, two other pairs were taken at $\chi=0.125$ (pair C, D) and $\chi=0.18$ (pair E, F) and used to calculate the constants and the predicted values of the critical concentrations at $\chi=0.05$ (pair A, B). The results are shown in Table II. As can be seen, the values calculated for the critical concentrations at $\chi=0.05$ are rather insensitive to the pairs of data points selected for the determination of the two system-dependent parameters.

\section{APPLICATION IN TERMS OF A THEORETICAL FRICTION FACTOR}

In practice experimental data for the friction factor will not always be available. So it would seem that the method

TABLE II. Predicted critical concentrations $\phi_{A}$ and $\phi_{B}$ when different pairs of data points (A,B; C,D; and E,F) are used for the determination of the constants $K_{b 1}$ and $K_{b 2}$.

\begin{tabular}{lcccc}
\hline \hline$\chi$ & Point 1 & Point 2 & Predicted $\phi_{A}$ & Predicted $\phi_{B}$ \\
\hline 0.05 & $\phi_{A}=0.9$ & $\phi_{B}=0.31$ & 0.9 & 0.31 \\
0.125 & $\phi_{C}=0.85$ & $\phi_{D}=0.375$ & 0.87 & 0.335 \\
0.18 & $\phi_{E}=0.82$ & $\phi_{F}=0.37$ & 0.86 & 0.34 \\
\hline \hline
\end{tabular}




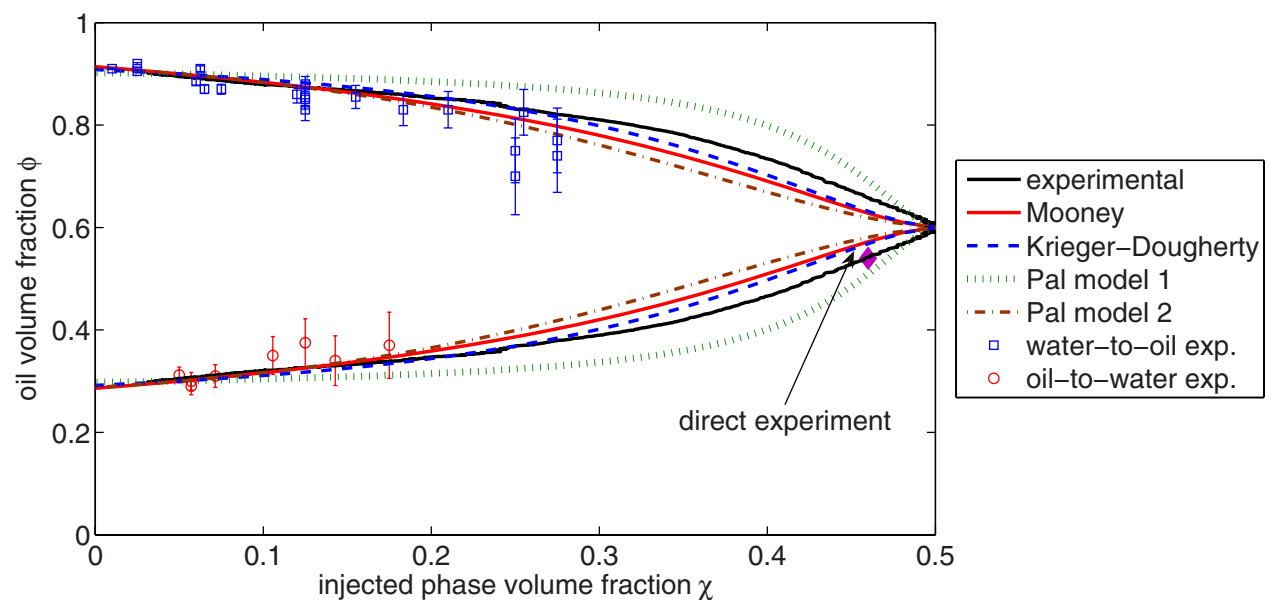

FIG. 9. (Color online) The oil volume fraction $\phi$ as a function of the injected phase volume fraction $\chi$ at the boundaries of the ambivalence region. The circles and squares indicate the experimental data points. The solid curves represent the values, earlier shown in Fig. 8, calculated from the Ginzburg-Landau model when the system-dependent constants are determined from the experimental friction factor $f$. The other curves represent the calculated values when the system-dependent constants are determined from the friction factor estimated with the aid of Eqs. (8)-(12).

presented in the last section can then not be applied. However, there is a solution to this problem. In practice experimental data on the oil volume fraction $\phi$ at inversion are often available and these data can be used to predict the friction factor $f$ at inversion by applying an empirical correlation between $f$ and $\phi$.

Assuming a homogeneous dispersed flow we can calculate the friction factor by applying the Blasius correlation $\left(2000<\operatorname{Re}<10^{5}\right)$

$$
f=\frac{0.316}{\operatorname{Re}^{0.25}}
$$

The effective viscosity $\eta$ in the Reynolds number $(\mathrm{Re})$ can be calculated by one of the following correlations:

(i) Mooney equation [30],

$$
\eta=\exp \left(\frac{2.5 \phi}{1-k \phi}\right)
$$

with $k=0.8$.

(ii) Krieger-Dougherty equation,

$$
\eta=\eta_{0}\left(1-\frac{\phi}{\phi_{c}}\right)^{-5 / 2 \phi_{c}} \text {. }
$$

(iii) Pal model 1 [31],

$$
\eta\left(\frac{2 \eta+5 K}{2+5 K}\right)^{3 / 2}=\exp \left(\frac{2.5 \phi}{1-\phi / \phi_{c}}\right) .
$$

(iv) Pal model 2 [31],

$$
\eta\left(\frac{2 \eta+5 K}{2+5 K}\right)^{3 / 2}=\exp \left(1-\frac{\phi}{\phi_{c}}\right)^{-2.5 \phi_{c}} .
$$

In Eqs. (10)-(12) $\phi_{c}$ is the closed-packing oil volume fraction. Extrapolating our water-to-oil experiments reported in [18] we find $\phi_{c}=0.95 . K$ is the ratio between the oil viscosity and the water viscosity. Applying one of these correlations to calculate $\eta$ it is possible to calculate Re, when also the mixture velocity and the effective density are known. Finally the friction factor $f$ is calculated from Re by means of Eq. (8). [By using Eq. (8) there is an influence of the mixture velocity on the friction factor and, therefore, also on the predicted width of the ambivalence region. However, as most of the experiments were performed in a rather narrow region of mixture velocities, this predicted influence is almost negligible. This is in agreement with the experimental data.] Then it is possible again to apply the method of the preceding section for predicting the ambivalence region. The results are shown in Fig. 9. The same two points as in the preceding section have been used to determine the constants in Eqs. (6) and (7). All models, except the Pal model 1, represent the boundaries of the ambivalence region rather well.

\section{DISCUSSION}

As can be seen from Figs. 8 and 9, a Ginzburg-Landau model can be used to describe phase inversion in an oilwater mixture. This is due to the close similarity between the hysteresis region for first-order phase transitions and the ambivalence region for phase inversion between two liquids in a dispersed flow.

According to our measurements [18], the ambivalence region is unique for a specific water-oil mixture. For such a mixture the ambivalence region depends on the injected phase volume fraction. It is independent of the Reynolds, Froude, and Weber numbers and also independent of the injection rate, as long as the mixture velocity is large enough. With our method the ambivalence region can be fully determined by carrying out only two experiments. Of course, when the oil type is changed or when a surfactant is added, the ambivalence region will change and two new experiments need to be performed. 


\section{ACKNOWLEDGMENTS}

The authors are indebted to Shell International Exploration and Production and to the Foundation for Fundamental Research on Matter (FOM) for the financial support to this project. They also thank R. V. A. Oliemans, J. Westerweel, and R. Delfos of Delft University of Technology for their continuous support to our research. Some helpful comments from M. E. Fisher and T. R. Kirkpatrick are also gratefully acknowledged.
[1] P. Becher, Emulsions: Theory and Practice (Oxford University Press, Oxford, 2001).

[2] L. Y. Yeo, O. K. Matar, E. S. Perez de Ortiz, and G. F. Hewitt, Multiphase Sci. Technol. 12, 51 (2000).

[3] M. Arashmid and G. V. Jeffreys, AIChE J. 26, 51 (1980).

[4] G. E. J. Vaessen, M. Visschers, and H. N. Stein, Langmuir 12, 875 (1996).

[5] L. Y. Yeo, O. K. Matar, E. S. Perez de Ortiz, and G. F. Hewitt, Chem. Eng. Sci. 57, 1069 (2002).

[6] N. Brauner and A. Ullmann, Int. J. Multiphase Flow 28, 1177 (2002).

[7] F. Groeneweg, W. G. M. Agterof, P. Jaeger, J. J. M. Janssen, J. A. Wieringa, and J. K. Klahn, Chem. Eng. Res. Des. 76, 55 (1998).

[8] K. B. Deshpande and S. Kumar, Chem. Eng. Sci. 58, 3829 (2003).

[9] I. Mira, N. Zambrano, E. Tyrode, L. Márquez, A. A. Peña, A. Pizzino, and J. -L. Salager, Ind. Eng. Chem. Res. 42, 57 (2003).

[10] E. Tyrode, I. Mira, L. Zambrano, L. Márquez, M. RondónGozalez, and J.-L. Salager, Ind. Eng. Chem. Res. 42, 4311 (2003).

[11] J. A. Quinn and D. B. Sigloh, Can. J. Chem. Eng. 41, 15 (1963).

[12] E. Tyrode, J. Allouche, L. Choplin, and J. -L. Salager, Ind. Eng. Chem. Res. 44, 67 (2005).

[13] L. Liu, O. K. Matar, C. J. Lawrence, and G. F. Hewitt, Chem. Eng. Sci. 61, 4007 (2006).

[14] R. Pal, AIChE J. 39, 1754 (1993).

[15] M. Nädler and D. Mewes, Int. J. Multiphase Flow 23, 55 (1997).
[16] K. Ioannou, O. J. Nydal, and P. Angeli, Exp. Therm. Fluid Sci. 29, 331 (2005).

[17] D. Chakrabarti, G. Das, and P. K. Das, AIChE J. 52, 3668 (2006).

[18] K. Piela, R. Delfos, G. Ooms, J. Westerweel, R. V. A. Oliemans, and R. F. Mudde, Int. J. Multiphase Flow 32, 1087 (2006).

[19] K. Piela, R. Delfos, G. Ooms, J. Westerweel, and R.V. A. Oliemans, Int. J. Multiphase Flow 34, 665 (2008).

[20] P. Chaikin and T. Lubensky, Principles of Condensed Matter Physics (Cambridge University Press, Cambridge, 1995).

[21] N. Brauner, Int. J. Multiphase Flow 27, 885 (2001).

[22] M. Luban, in Phase Transitions and Critical Phenomena, edited by C. Domb and M. Green (Academic, New York, 1976), Vol. 5a, p. 35.

[23] G. Baker, in Bifurcation Phenomena in Mathematical Physics and Related Topics, edited by C. Bardos and D. Bessis (Reidel, Dordrecht, 1980), p. 91.

[24] M. A. Anisimov, S. B. Kiselev, J. V. Sengers, and S. Tang, Physica A 188, 487 (1992).

[25] J. Wang and M. A. Anisimov, Phys. Rev. E 75, 051107 (2007).

[26] Y. C. Kim, M. E. Fisher, and G. Orkoulas, Phys. Rev. E 67, 061506 (2003).

[27] J. Wang, C. A. Cerdereiña, M. A. Anisimov, and J. V. Sengers, Phys. Rev. E 77, 031127 (2008).

[28] R. Gilmore, Catastrophe Theory for Scientists and Engineers (Wiley, New York, 1981).

[29] E. Dickinson, J. Colloid Interface Sci. 84, 284 (1981).

[30] M. Mooney, J. Colloid Sci. 6, 162 (1951).

[31] R. Pal, J. Colloid Interface Sci. 225, 359 (2000). 